\title{
Ultrasensitive Magnetic Field Sensing Based on Refractive-Index-Matched Coupling
}

\author{
Jie Rao ${ }^{1}$, Shengli Pu ${ }^{1,2, * \mathbb{D}}$, Tianjun Yao ${ }^{1}$ and Delong Su ${ }^{1}$ \\ 1 College of Science, University of Shanghai for Science and Technology, Shanghai 200093, China; \\ 152251908@st.usst.edu.cn (J.R.); 162281924@st.usst.edu.cn (T.Y.); 142231886@st.usst.edu.cn (D.S.) \\ 2 Shanghai Key Laboratory of Modern Optical System, University of Shanghai for Science and Technology, \\ Shanghai 200093, China \\ * Correspondence: shlpu@usst.edu.cn; Tel.: +86-21-6566-6454
}

Received: 22 May 2017; Accepted: 3 July 2017; Published: 7 July 2017

\begin{abstract}
An ultrasensitive magnetic field sensor is proposed and investigated experimentally. The no-core fiber is fusion-spliced between two pieces of single-mode fibers and then immersed in magnetic fluid with an appropriate value of refractive index. Under the refractive-index-matched coupling condition, the guided mode becomes leaky and a coupling wavelength dip in the transmission spectrum of the structure is observed. The coupling wavelength dip is extremely sensitive to the ambient environment. The excellent sensitivity to the refractive index is measured to be $116.681 \mu \mathrm{m} / \mathrm{RIU}$ (refractive index unit) in the refractive index range of 1.45691-1.45926. For the as-fabricated sensors, the highest magnetic field sensing sensitivities of 6.33 and $1.83 \mathrm{~nm} / \mathrm{mT}$ are achieved at low and high fields, respectively. The sensitivity is considerably enhanced compared with those of previously designed, similar structures.
\end{abstract}

Keywords: magnetic field sensor; magnetic fluid; singlemode-no-core-singlemode fiber structures; refractive-index-matched coupling

\section{Introduction}

Magnetic fluid (MF) is a novel kind of functional material, which presents solid-magneticmaterial-like magnetism and liquid-like fluidity. It consists of magnetic nanoparticles dispersed in a suitable liquid carrier. The magnetic nanoparticles are coated with surfactant to form a stable colloidal system [1]. The microstructures within MF are diverse under the external applied magnetic field. This will lead to many unique optical properties of MF under an applied magnetic field, such as tunable refractive index (RI), tunable birefringence, and tunable transmittance [2]. Until now, many optical devices and applications based on MF have been proposed and demonstrated, for example, optical gratings [3,4], optical switches [5], magnetic field sensors [1,6-16], tunable filters [17], tunable slow light [18], and couplers [19].

Recently, the singlemode-multimode-singlemode (SMS) or singlemode-no-core-singlemode (SNS) fiber structures have been designed to sense temperature, RI, chemical concentration, strain and magnetic field $[1,6,20-23]$. They are low-cost and easy to fabricate. The fundamental sensing principle is the multimode interference within the multimode fiber or no-core fiber (NCF). The sensitivity of these structures depends on the difference of response to ambient variation between the involved modes. On the other hand, refractive-index-matched coupling (RIMC) is attractive for widely tunable filtering and ultrasensitive sensing applications [24-27]. The fundamental principle is based on the material dispersion effect.

In this work, an MF with an appropriate RI value is utilized to cover the SNS fiber structure to realize magnetic field sensing with ultrahigh sensitivity. Different from the conventional SMS/SNS 
fiber structures, the sensing principle of this work is based on RIMC. At RIMC, the guided modes within the NCF become leaky, which yield a coupling wavelength dip (CWD) in the transmission spectrum. The CWD will shift significantly, and even the ambient environment changes slightly. Therefore, the sensitivity of the proposed magnetic field sensor can be markedly enhanced.

\section{Sensor Structure and Sensing Principle}

Figure 1a shows the schematic diagram of the proposed sensor. A section of NCF with a diameter of $125 \mu \mathrm{m}$ is fusion spliced between two pieces of singlemode fibers. The whole structure is placed in a capillary tube, which is filled with MF. Both ends of the capillary tube are sealed with UV glue to prevent the MF from leaking and evaporating. Therefore, the cladding of the NCF is actually the ambient MF. The as-fabricated sensor is shown in Figure $1 \mathrm{~b}$.
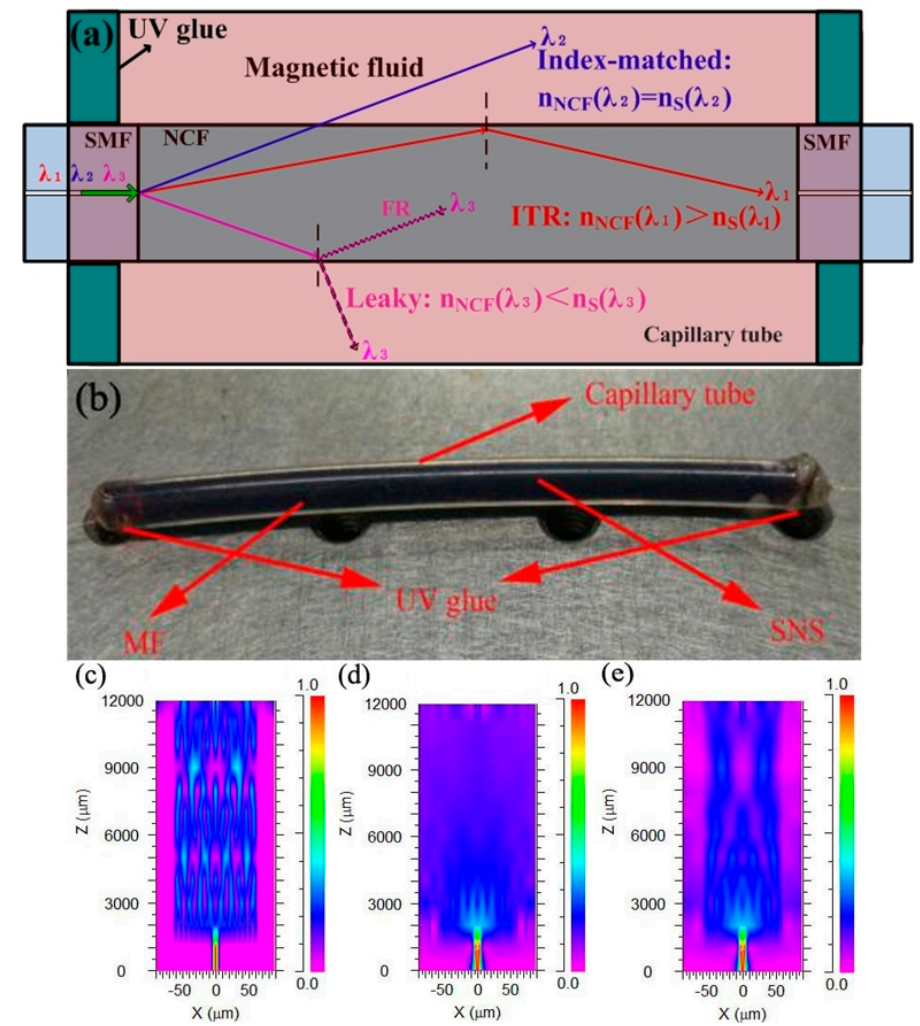

Figure 1. (a) Schematic of the proposed sensor; (b) picture of the as-fabricated sensor; (c) simulation of energy distribution within the no-core fiber (NCF) for the guided $\left(n_{N C F}\left(\lambda_{1}\right)>n_{S}\left(\lambda_{1}\right)\right)$; (d) index-matched $\left(n_{N C F}\left(\lambda_{2}\right)=n_{S}\left(\lambda_{2}\right)\right)$ and (e) leaky $\left(n_{N C F}\left(\lambda_{3}\right)<n_{S}\left(\lambda_{3}\right)\right)$ cases, respectively.

When the incident light is broadband and propagates within the NCF, there are three possible cases depending on the relative relationship between the RI of NCF and that of MF: guided, index-matched, and leaky (see Figure 1a). Due to the different dispersion properties of NCF and MF, their dispersion profiles may intersect at a certain wavelength range. The guided condition means that the internal total reflection (ITR) happens, i.e., $n_{N C F}\left(\lambda_{1}\right)>n_{S}\left(\lambda_{1}\right)$, where $n_{N C F}\left(\lambda_{1}\right)$ and $n_{S}\left(\lambda_{1}\right)$ are the RIs of NCF and MF at $\lambda_{1}$, respectively. At a specific wavelength (for example $\left.\lambda_{2}\right), n_{N C F}\left(\lambda_{2}\right)=n_{S}\left(\lambda_{2}\right)$ and the index-matched condition is satisfied. This is the RIMC, which can cause a high loss at this specific wavelength. The leaky case implies $n_{N C F}\left(\lambda_{3}\right)<n_{S}\left(\lambda_{3}\right)$ and the Fresnel reflection (FR) will happen at this wavelength. Figure 1c-e) displays the simulations of energy distribution within the NCF for the three cases, respectively. It is clear from Figure 1c-e that the light is effectively confined within the NCF when $n_{N C F}\left(\lambda_{1}\right)>n_{S}\left(\lambda_{1}\right)$. Strong interference is obvious. Under the index-matched condition $\left(n_{N C F}\left(\lambda_{2}\right)=n_{S}\left(\lambda_{2}\right)\right)$, the energy leaks out of NCF along with the light propagation. Meanwhile, for 
the FR case $\left(n_{N C F}\left(\lambda_{3}\right)<n_{S}\left(\lambda_{3}\right)\right)$, part of energy returns to the fiber. Then, weak light is observed within the NCF as shown in Figure 1e.

The CWD is related with the index-match between the NCF and the surrounding media (MF) at a specific wavelength. The RI of the MF changes with the external magnetic field, but that of the NCF is independent of magnetic field. So, the index-matched wavelength will shift with the magnetic field. Due to the low dispersion of the NCF and MF, i.e., small slopes of the dispersion profiles, the index-matched wavelength will shift significantly, and even the ambient environment varies slightly. Thus, the magnetic field sensing with ultrahigh sensitivity can be realized through observing the shift of the CWD.

\section{Experimental Results and Discussion}

The schematic of the experimental setup for investing the sensing properties is shown in Figure 2. Light from the supercontinuum broadband source (SBS, Wuhan Yangtze Soton Laser Co., Ltd., Wuhan, China) is launched into the sensing structure and the output light is monitored and analyzed by an optical spectrum analyzer (OSA, Yokogawa AQ6370C, Tokyo, Japan). The magnetic field strength is adjusted by changing the magnitude of the supply current and calibrated by a gauss meter. The magnetic field direction is perpendicular to the optical fiber axis. During our experiments, the ambient temperature is kept constant.

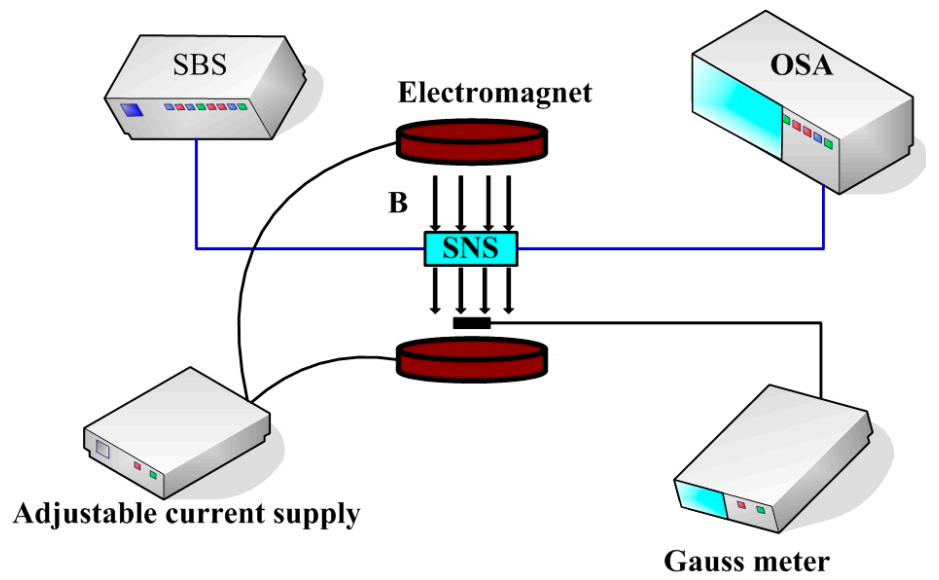

Figure 2. Schematic diagram of the experimental setup for investigating the sensing properties.

The NCF with a diameter of $125 \mu \mathrm{m}$ was provided by Yangtze Optical Fibre and Cable Joint Stock Limited Company (Wuhan, China). The RI of the oil-based MF (EXP08103, Ferrotec, Chiba, Japan) with volume fraction of $5.62 \%$ used in this work is measured to be 1.56403 by a refractometer (A670, Hanon, Jinan, China). For the easy occurrence of the abovementioned three cases, the initial MF is diluted with ethyl oleate to around $0.4 \%$ volume fraction, whose RI approaches that of the NCF. The diluted MFs with RIs of 1.45468, 1.45691, 1.45786, and 1.45926 (measured by the same refractometer) are obtained through finely tuning the volume fraction further, which are then utilized to fabricate the proposed sensing structures. The corresponding transmission spectra are shown in Figure $3 a$. Figure 3a indicates that the spectra for the MF-covered SNS structures is much smoother than that without the MF. This may be due to the RI of the MF approaching that of the NCF, which leads to the weakly guided case. More high-order modes radiate into the MF and become lossy, which weakens the modal interference effect. For clarity, the spectra at short and long wavelength regimes are replotted in Figure $3 b, c$ respectively. Figure $3 b$ shows that the spectrum red-shifts with the RI of the MF (see the red arrow). This is qualitatively consistent with that in [6]. Therefore, the mode interference dominates the spectrum at the short wavelength regime. The total wavelength shift is $40.2 \mathrm{~nm}$. For the RI ranging 
from 1.45468 to 1.45926 , the obtained wavelength shift is $13.8 \mathrm{~nm}$ and the corresponding average RI sensing sensitivity is $3013 \mathrm{~nm} / \mathrm{RIU}$ (refractive index unit).
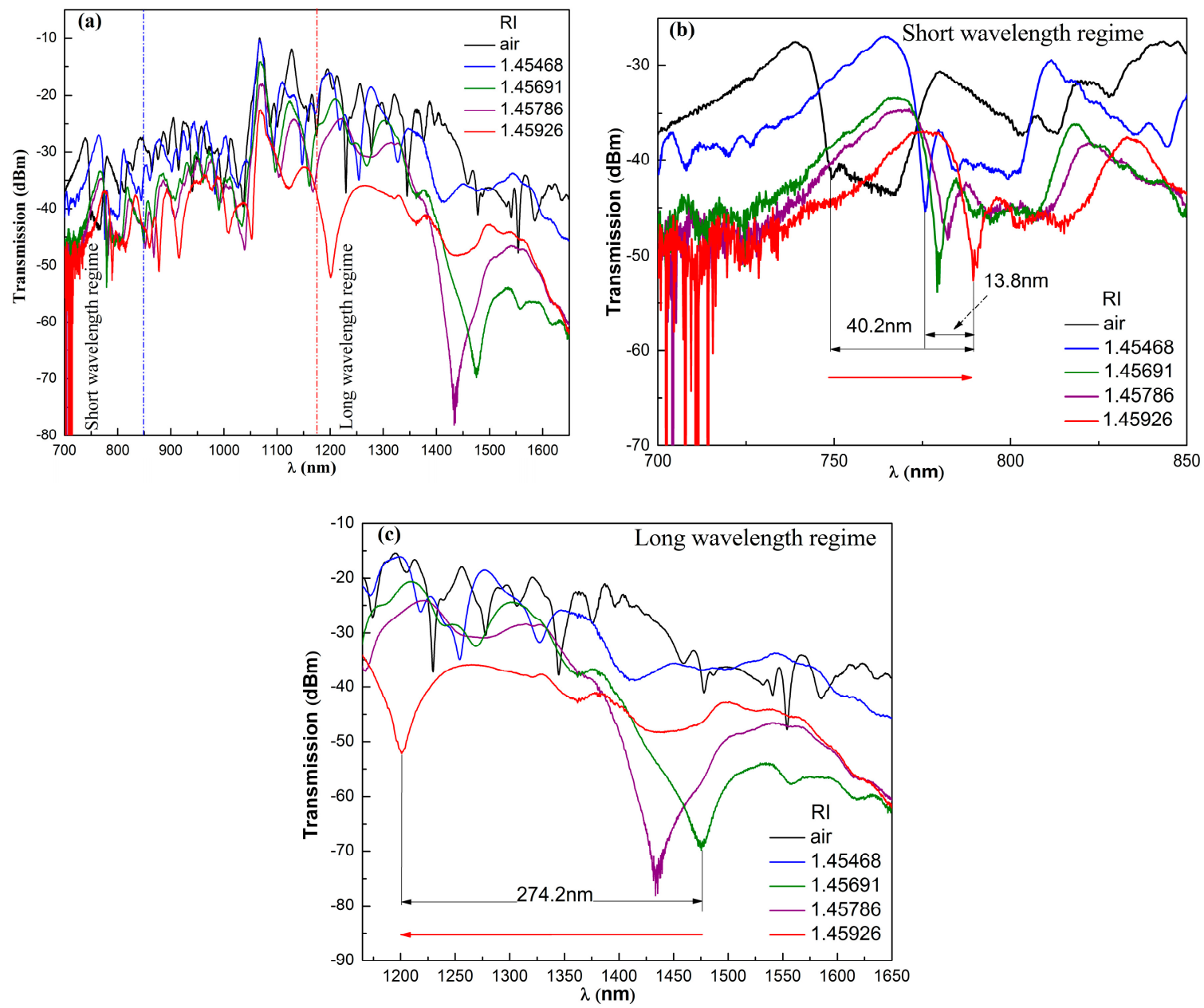

Figure 3. (a) Transmission spectra of the as-fabricated sensing structures, (b) the corresponding spectra at the short wavelength regime and (c) at the long wavelength regime.

At the long wavelength regime, there is a newly emerging dip wavelength occurring around $1480 \mathrm{~nm}$ when the RI of the MF is increased to 1.45691 (see Figure 3c). When the RI of the MF further increases, the newly emerging dip wavelength shifts toward the short wavelength side (see the red arrow in Figure 3c), which is opposite to the mode interference effect at the short wavelength regime. This newly emerging dip wavelength corresponds to the CWD. At this specific wavelength, the RI of the MF equals that of the NCF. To illustrate the shift of the CWD explicitly, Figure 4 schematically plots the dispersion profiles of the NCF and MFs with different RIs. The intersection point between the dispersion profiles of the NCF and MF corresponds to the CWD. Figure 4 clearly indicates that the intersection point shifts toward the short wavelength side as the RI of the MF increases. This is in agreement with the experimental results shown in Figure 3c. 


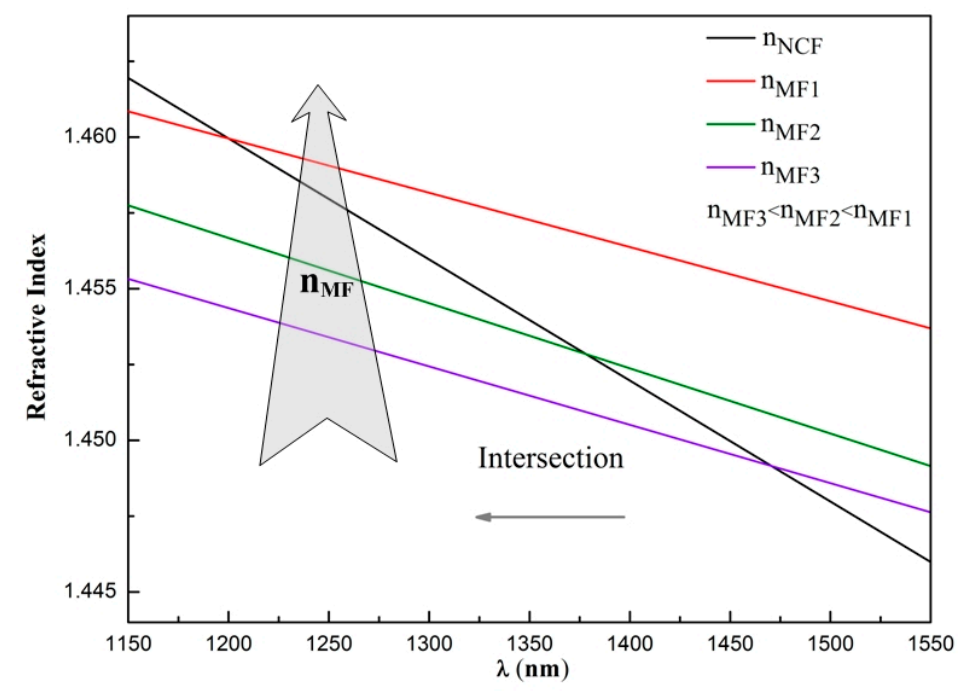

Figure 4. Dispersion profiles of the NCF and magnetic fluids (MFs) with different refractive indices (RIs).

At the long wavelength regime, the total wavelength shift is $274.2 \mathrm{~nm}$ in the range of 1.45691-1.45926, which corresponds to an average RI sensing sensitivity of $116.681 \mu \mathrm{m} / \mathrm{RIU}$. Hence, the sensitivity based on the RIMC (leak of guided modes) is around 39 times higher than that based on mode interference. Thus, the proposed structure can be employed to realize a magnetic field sensor with ultrahigh sensitivity through interrogating the CWD.

In order to investigate the magnetic field sensing characteristics of the proposed structure in detail, the MF with an RI of 1.45786 is first employed, and two structures consisting of NCF with lengths of 2.5 and $3.5 \mathrm{~cm}$ are fabricated. The experimental results are shown in Figure 5. Figure 5a,b display that the CWDs (denoted as C) move to the short wavelength side with the magnetic field (denoted as B), which is contributed to the increase of the RI of the MF with the magnetic field [28]. To be more explicit, the shift of the CWDs labeled in Figure 5a,b with the magnetic field is replotted in Figure 5c. Figure 5c indicates that the CWDs change significantly with the magnetic field under the low field region $(\leq 6 \mathrm{mT})$. The corresponding sensitivities are 5.49 and $5.62 \mathrm{~nm} / \mathrm{mT}$, respectively. As the magnetic field continues to increase, the shift becomes slight. This is due to the saturation magnetization of the MF at a relatively high magnetic field. The corresponding sensitivities at higher field regions (6-26 $\mathrm{mT}$ ) are 1.51 and $1.42 \mathrm{~nm} / \mathrm{mT}$, respectively. The experimental results indicate that the length of the NCF has a negligible effect on the sensitivity of the SNS structure.
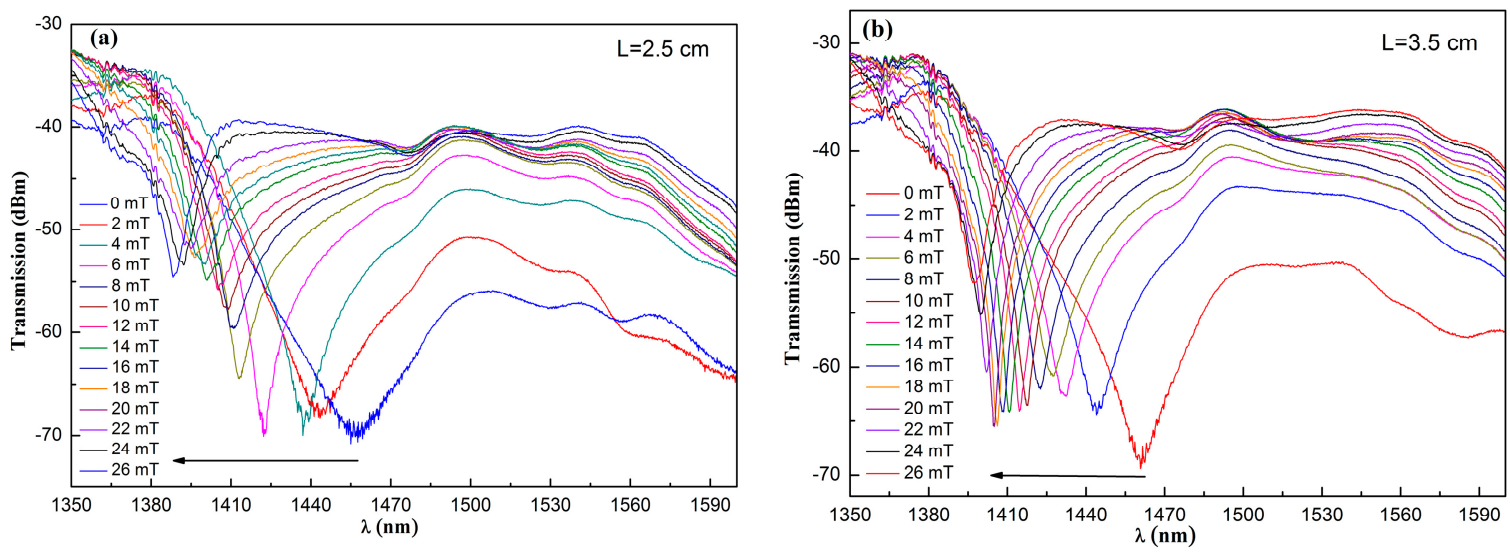

Figure 5. Cont. 


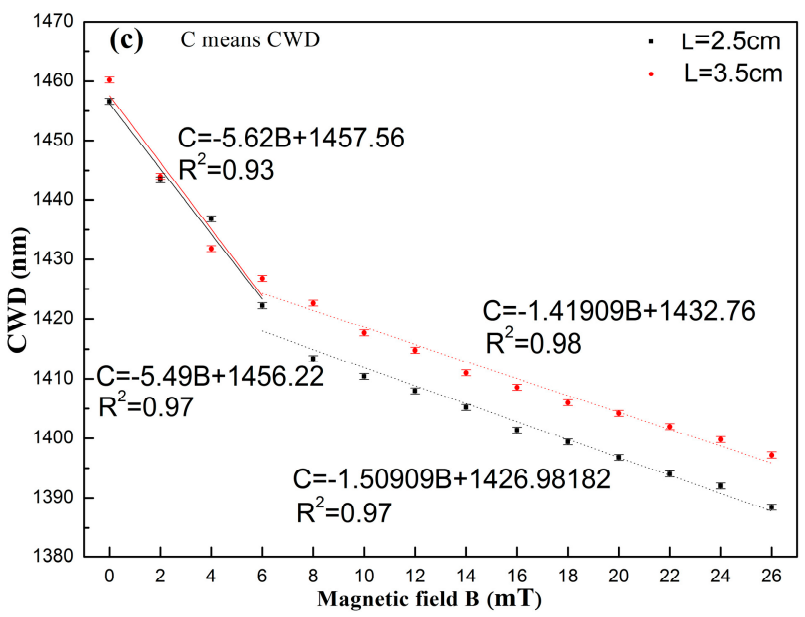

Figure 5. Transmission spectra of the sensing structures consisting of the NCF with lengths of (a) $2.5 \mathrm{~cm}$ and (b) $3.5 \mathrm{~cm}$; (c) the relationship between the coupling wavelength dip (CWD) and the magnetic field intensity.

Furthermore, another two SNS structures are fabricated employing MFs with an RI of 1.45691 and 1.45926 , respectively. The length of the NCF is kept at $3.5 \mathrm{~cm}$. The very similar experimental results are obtained and shown in Figure 6.
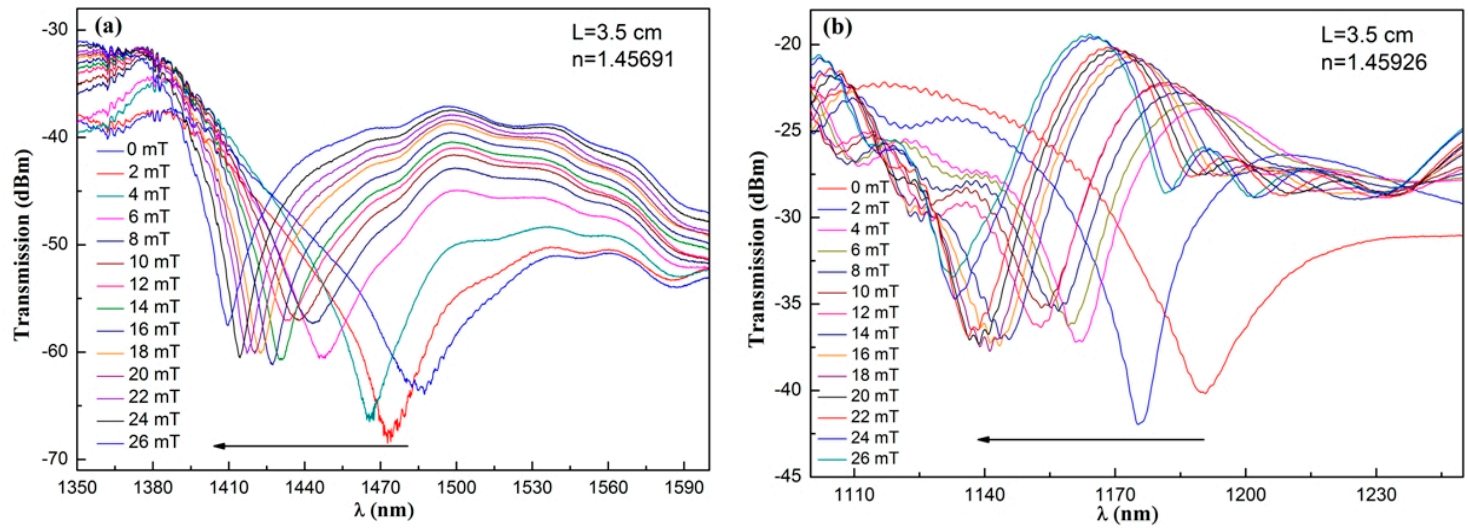

Figure 6. Transmission spectra of the sensing structures with the RI of the MF of (a) 1.45691 and (b) 1.45926. The length of the NCF is $3.5 \mathrm{~cm}$.

According to Figures 5 and 6, the relationship between the CWDs and the magnetic field for the three structures with the same length of NCF $(3.5 \mathrm{~cm})$ covered with different RIs of the MF (1.45691, 1.45786, and 1.45926) is plotted in Figure 7. From Figure 7, the sensitivities of the three structures are $6.33,5.62$, and $5.50 \mathrm{~nm} / \mathrm{mT}$ under the low magnetic field region $(\leq 6 \mathrm{mT})$, respectively. Under the high magnetic field $(6-26 \mathrm{mT})$, the decreased sensitivities of $1.83,1.42$, and $1.46 \mathrm{~nm} / \mathrm{mT}$ are obtained, respectively. The structure covered with the lowest RI of the MF (1.45691) has the highest sensitivity. This may be due to the dispersion profile difference between the MFs with different RIs. Then, the intersection point of the dispersion profile between the NCF and MF shifts differently for different structures under the same magnetic field change. 


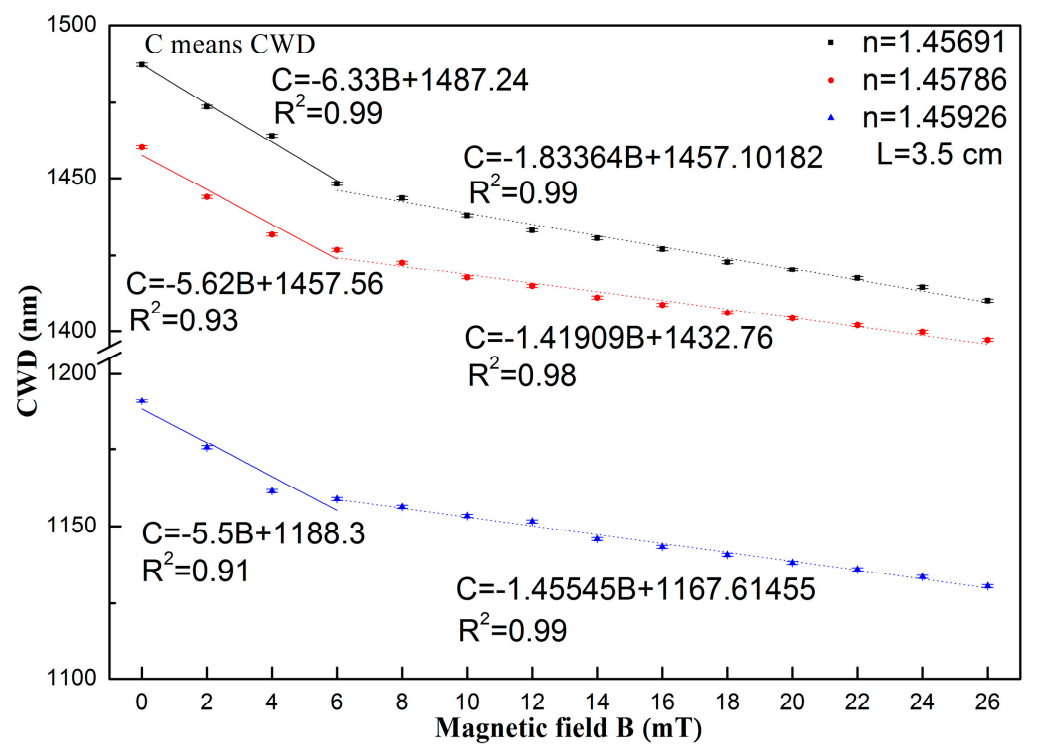

Figure 7. Relationship between the CWD and the magnetic field intensity for the sensing structures covered with an MF of different RIs. The length of the NCF is $3.5 \mathrm{~cm}$.

Though the low concentration MF is utilized in this work, the sensitivity has been improved significantly compared with those of previously used similar structures, based on mode interference. The highest sensitivity of $6.33 \mathrm{~nm} / \mathrm{mT}$ was achieved under the low magnetic field region $(\leq 6 \mathrm{mT})$, which is around 38 times and 7 times higher than those of the very similar SMS $(0.1686 \mathrm{~nm} / \mathrm{mT})$ and SNS $(0.905 \mathrm{~nm} / \mathrm{mT})$ structures based on the multimode interference effect $[1,6]$.

\section{Conclusions}

In conclusion, a novel magnetic field sensor based on MF and RIMC is proposed. The traditional SNS fiber structure is employed, but the sensing principle is totally different from the previously employed multimode interference. The length of the NCF has a negligible effect on the sensitivity of the SNS structure. For the as-fabricated structures, the highest sensitivities of 6.33 and $1.83 \mathrm{~nm} / \mathrm{mT}$ were achieved under the low magnetic field $(\leq 6 \mathrm{mT})$ and high magnetic field $(6-26 \mathrm{mT})$ regions, respectively. The maximum sensitivity in this work is considerably enhanced compared with those of the previous designed, similar structures. By choosing an MF with high saturation magnetization and designing the initial RI of the MF (under zero magnetic field) with an appropriate value, the sensitivity and sensing range of the proposed magnetic field sensor can be further improved.

Acknowledgments: This research is supported by National Natural Science Foundation of China (Grant No. 61675132), Shanghai "Shuguang Program" (Grant No. 16SG40), Shanghai Talent Development Fund (Grant No. 201529), Natural Science Foundation of Shanghai (Grant No. 13ZR1427400), Shanghai Key Laboratory of Specialty Fiber Optics and Optical Access Networks (Grant No. SKLSFO2014-05), and Hujiang Foundation of China (Grant No. B14004).

Author Contributions: Jie Rao conceived, designed and performed the experiments; Jie Rao and Shengli Pu analyzed the data, and co-wrote the manuscript; Shengli Pu planned and supervised the research, and gave suggestions at all stages. Tianjun Yao and Delong Su contributed to the simulations. All authors contributed to the discussions and reviewed the manuscript.

Conflicts of Interest: The authors declare no conflict of interest.

\section{References}

1. Wang, H.; Pu, S.; Wang, N.; Dong, S.; Huang, J. Magnetic field sensing based on singlemodemultimode-singlemode fiber structures using magnetic fluids as cladding. Opt. Lett. 2013, 38, 3765-3768. [CrossRef] [PubMed] 
2. Zhao, Y.; Lv, R.; Zhang, Y.; Wang, Q. Novel optical devices based on the transmission properties of magnetic fluid and their characteristics. Opt. Lasers Eng. 2012, 50, 1177-1184. [CrossRef]

3. Pu, S.; Chen, X.; Chen, L.; Liao, W.; Chen, Y.; Xia, Y. Tunable magnetic fluid grating by applying a magnetic field. Appl. Phys. Lett. 2005, 87, 021901. [CrossRef]

4. Zaibudeen, A.W.; Philip, J. Thermally tunable grating using thermo-responsive magnetic fluid. Opt. Mater. 2017, 66, 117-121. [CrossRef]

5. Horng, H.E.; Chen, C.S.; Fang, K.L.; Yang, S.Y.; Chieh, J.J.; Hong, C.-Y.; Yang, H.C. Tunable optical switch using magnetic fluids. Appl. Phys. Lett. 2004, 85, 5592-5594. [CrossRef]

6. Chen, Y.; Han, Q.; Liu, T.; Lan, X.; Xiao, H. Optical fiber magnetic field sensor based on single-mode-multimode-single-mode structure and magnetic fluid. Opt. Lett. 2013, 38, 3999-4001. [CrossRef] [PubMed]

7. Xia, J.; Wang, F.; Luo, H.; Wang, Q.; Xiong, S. A magnetic field sensor based on a magnetic fluid-filled FP-FBG structure. Sensors 2016, 16, 620. [CrossRef] [PubMed]

8. Wu, J.; Miao, Y.; Song, B.; Lin, W.; Zhang, H.; Zhang, K.; Liu, B.; Yao, J. Low temperature sensitive intensity-interrogated magnetic field sensor based on modal interference in thin-core fiber and magnetic fluid. Appl. Phys. Lett. 2014, 104, 252402. [CrossRef]

9. Shi, J.; Wang, Y.; Xu, D.; Su, G.; Zhang, H.; Feng, J.; Yan, C.; Fu, S.; Yao, J. Remote magnetic field sensor based on intracavity absorption of evanescent field. IEEE Photonics J. 2016, 8, 6801607. [CrossRef]

10. Chen, Y.; Han, Q.; Yan, W.; Yao, Y.; Liu, T. Magnetic-fluid-coated photonic crystal fiber and FBG for magnetic field and temperature sensing. IEEE Photonics Technol. Lett. 2016, 28, 2665-2668. [CrossRef]

11. Bai, X.; Yuan, J.; Gu, J.; Wang, S.; Zhao, Y.; Pu, S.; Zeng, X. Magnetic field sensor using fiber ring cavity laser based on magnetic fluid. IEEE Photonics Technol. Lett. 2016, 28, 115-118. [CrossRef]

12. Liu, H.; Zhang, H.; Liu, B.; Song, B.; Wu, J.; Lin, L. Ultra-sensitive magnetic field sensor with resolved temperature cross-sensitivity employing microfiber-assisted modal interferometer integrated with magnetic fluids. Appl. Phys. Lett. 2016, 109, 042402. [CrossRef]

13. Mao, L.; Pu, S.; Su, D.; Wang, Z.; Zeng, X.; Lahoubi, M. Magnetic field sensor based on cascaded microfiber coupler with magnetic fluid. J. Appl. Phys. 2016, 120, 093192. [CrossRef]

14. Zhang, Z.; Guo, T.; Zhang, X.; Xu, J.; Xie, W.; Nie, M.; Wu, Q.; Guan, B.-O.; Albert, J. Plasmonic fiber-optic vector magnetometer. Appl. Phys. Lett. 2016, 108, 101105. [CrossRef]

15. Luo, L.; Pu, S.; Tang, J.; Zeng, X.; Lahoubi, M. Highly sensitive magnetic field sensor based on microfiber coupler with magnetic fluid. Appl. Phys. Lett. 2015, 106, 193507. [CrossRef]

16. Candiani, A.; Argyros, A.; Leon-Saval, S.G.; Lwin, R.; Selleri, S.; Pissadakis, S. A loss-based magnetic field sensor implemented in a ferrofluid infiltrated microstructured polymer optical fiber. Appl. Phys. Lett. 2014, 104, 111106. [CrossRef]

17. Lin, W.; Liu, B.; Zhang, H.; Song, B.; Yan, D.; Miao, Y.; Liu, H.; Liu, Y. Laser-induced thermal effect for tunable filter employing ferrofluid and fiber taper coupler. IEEE Photonics Technol. Lett. 2015, 27, 2339-2342. [CrossRef]

18. Pu, S.; Dong, S.; Huang, J. Tunable slow light based on magnetic-fluid-infiltrated photonic crystal waveguides. J. Opt. 2014, 16, 045102. [CrossRef]

19. Tagoudi, E.; Milenko, K.; Pissadakis, S. Intercore coupling effects in multicore optical fiber tapers using magnetic fluid out-claddings. J. Light. Technol. 2016, 34, 5561-5565. [CrossRef]

20. Wu, Q.; Semenova, Y.; Hatta, A.; Wang, P.; Farrell, G. Bent SMS fiber structure for temperature measurement. Electron. Lett. 2010, 46, 1129-1130. [CrossRef]

21. Wang, Q.; Farrell, G. All-fiber multimode-interference-based refractometer sensor: Proposal and design. Opt. Lett. 2006, 31, 317-319. [CrossRef] [PubMed]

22. Lan, X.; Huang, J.; Han, Q.; Wei, T.; Gao, Z.; Jiang, H.; Dong, J.; Xiao, H. Fiber ring laser interrogated zeolite-coated singlemode-multimode-singlemode structure for trace chemical detection. Opt. Lett. 2012, 37, 1998-2000. [CrossRef] [PubMed]

23. Tripathi, S.M.; Kumar, A.; Varshney, R.K.; Kumar, Y.B.P.; Marin, E.; Meunier, J.-P. Strain and temperature sensing characteristics of single-mode-multimode-single-mode structures. J. Lightwave Technol. 2009, 27, 2348-2356. [CrossRef] 
24. Lee, C.-L.; Lin, K.-H.; Lin, Y.Y.; Hsu, J.-M. Widely tunable and ultrasensitive leaky-guided multimode fiber interferometer based on refractive-index-matched coupling. Opt. Lett. 2012, 37, 302-305. [CrossRef] [PubMed]

25. Bhowmik, T.; Maity, A. Design and analysis of broadband single-mode photonic crystal fiber for transmission windows of the telecom wavelengths. Opt.-Int. J. Light Electron Opt. 2017, 139, 366-372. [CrossRef]

26. Hou, Y.; Fan, F.; Zhang, H.; Wang, X.-H.; Chang, S.-J. Terahertz single-polarization single-mode hollow-core fiber based on index-matching coupling. IEEE Photonics Technol. Lett. 2012, 24, 637-639. [CrossRef]

27. Sun, B.; Chen, M.-Y.; Zhang, Y.-K.; Yang, J.-C. Design of refractive index sensors based on the wavelength-selective resonant coupling phenomenon in dual-core photonic crystal fibers. J. Biomed. Opt. 2012, 17, 037002. [CrossRef] [PubMed]

28. Hong, C.-Y.; Chieh, J.-J.; Yang, S.-Y.; Yang, H.-C.; Horng, H.-E. Simultaneous identification of the low-field-induced tiny variation of complex refractive index for anisotropic and opaque magnetic-fluid thin film by a stable heterodyne Mach-Zehnder interferometer. Appl. Opt. 2009, 48, 5604-5611. [CrossRef] [PubMed]

(C) 2017 by the authors. Licensee MDPI, Basel, Switzerland. This article is an open access article distributed under the terms and conditions of the Creative Commons Attribution (CC BY) license (http:/ / creativecommons.org/licenses/by/4.0/). 